\title{
Épandages de lisiers : conséquences sur le phosphore biodisponible et sur la concentration de quelques cations dans la solution du sol
}

\author{
JC Fardeau ${ }^{1 *}, \mathrm{~J} \mathrm{Martinez}^{2}$ \\ 1 DEVM, CEA, Cadarache, F-13108 Saint-Paut-lès-Durance cedex; \\ 2 Cemagref, 17, avenue de Cucillé, F-35044 Rennes cedex, France
}

(Reçu le 27 septembre 1995; accepté le 3 avril 1996)

\begin{abstract}
Résumé - Les variations pluriannuelles de biodisponibilité du phosphore et de quelques cations présents dans la solution du sol ont été analysées dans un dispositif expérimental de plein champ mis en place pour transformer l'azote organique du lisier en azote moléculaire. Le dispositif est implanté en Bretagne sur un sol acide. Il comporte une parcelle témoin ne recevant pas de lisier et quatre sous-parcelles recevant du lisier ; l'ensemble est cultivé de Lolium perenne. Les apports annuels de lisier ont été en moyenne de $1000 \mathrm{~m}^{3} \mathrm{ha}^{-1} \mathrm{an}^{-1}$ et ceux de phosphore associé aux lisier ont été de $1400 \mathrm{kgP} \mathrm{ha}^{-1} \mathrm{an}^{-1}$. Des échantillons de terre ont été prélevés chaque année avant le début de chaque campagne d'épandage dans les horizons $0-20 \mathrm{~cm}, 20-40 \mathrm{~cm}$ et $40-60 \mathrm{~cm}$ des cinq parcelles. La méthode des cinétiques d'échanges isotopiques et la méthode Dyer ont été utilisées pour caractériser le phosphore biodisponible. Les résultats essentiels sont les suivants : i) à l'échelle pluriannuelle, et en situations d'épandages répétitifs, le phosphore présent dans le lisier peut être considéré comme aussi assimilable que celui des engrais phosphatés soluble-eau ; ii) la présence de matière organique dans le lisier contribue à diminuer le pouvoir fixateur de ce sol pour les ions phosphate ; iii) la méthode Dyer, généralement utilisée en France pour l'analyse du phosphore «assimilable» des sols non calcaires, extrait des formes de phosphore disponible par les plantes en l'état : iv) aux doses appliquées dans ce sol acide dont le pouvoir fixateur était initialement élevé, et plus élevé que la moyenne des sols de cette région, du phosphore a migré significativement au-delà de la couche labourée. Cependant l'accroissement du pouvoir fixateur avec la profondeur limitera généralement toute possibilité de migration des ions phosphate jusqu'aux nappes phréatiques.
\end{abstract}

lisier / phosphore biodisponible / P Dyer / échange isotopique / migration P / migration K

Summary - Pig slurry applications: effects on bioavailable phosphorus and cation concentration in soil solution. Bioavailability of soil phosphorus and cation concentration $\left(\mathrm{Ca}^{++}, \mathrm{Mg}^{++}, \mathrm{K}^{+}\right.$and $\left.\mathrm{Na}^{+}\right)$in the soil solution were analysed in soil samples taken from an experimental field designed to study the denitrification of nitrogen contained in pig slurry. The experimental set-up, located in Brittany (western France) on an acid soil, comprised a control plot that did not receive pig slurry, and four plots which all received pig slurry at the same rate. The experimental design was cropped with Lolium perenne. The mean rate of slurry application was about $1000 \mathrm{~m}^{3}$.year-1. ha-1 and of phosphorus 1 $400 \mathrm{kgP}_{\text {.year }}{ }^{-1}$.ha $\mathrm{a}^{-1}$. Soil samples were taken each year in three soil layers $0-20,20-40$ and $40-60 \mathrm{~cm}$ just before each first campaign of slurry application. The main results observed were the following: (i) on a yearly scale, phosphorus included in slurry must be considered to be as available as phosphorus derived from water-soluble $P$ fertilizers; (ii) 
organic matter in the slurry decreases the phosphate-fixing capacity of soils; as a consequence the risk of eutrophization of surface water is increased when soil particles containing available soil $P$ enter into contact with surface waters; (iii) the Dyer method with $2 \%$ citric acid extraction, currently used in France to analyse 'available soil $P$ ' in non calcareous soils, overestimates it by dissolving calcium/magnesium phytate in soils that recently received pig slurry; (iv) at the rates of application utilized in this experiment, even in this acid soil where the initial fixing capacity was high, a $P$ migration took place below the ploughed layer. However, the increase of P-fixing capacity with depth will generally limit the leaching of $P$ into ground water.

\section{pig slurry / bioavailable soil P / P Dyer / isotopic exchange / P migration / K migration}

\section{INTRODUCTION}

Le sol est en général le récepteur ultime du lisier de porc. Cette pratique était pleinement justifiée dans l'agriculture de subsistance : elle permettait de restituer au niveau de l'exploitation agricole, mais le plus souvent seulement sur certaines parcelles, la majorité des éléments nutritifs $N, P$ et $K$ exportés des sols, via la production végétale, quelques jours ou quelques mois auparavant pour assurer la nutrition des porcs et plus généralement de tous les animaux de la ferme (Pape, 1970). Le développement des élevages hors-sol, pour lesquels l'essentiel de l'alimentation est fréquemment originaire d'une région distincte de celle où sont engraissés les porcs, a profondément modifié les données du problème et surtout la solution possible pour que l'agriculteur puisse recycler son lisier (Sharpley et Withers, 1994). La dégradation croissante des eaux douces a conduit la Communauté européenne à adopter une directive destinée à protéger ces eaux contre la pollution par les nitrates à partir de sources agricoles ( $J O n^{\circ} \mathrm{L} 375 / 1$ du 12/12/91). Cette directive a été transposée en droit français par le décret $n^{\circ}$ 96-163 ( $\mathrm{JO}$ du 05/03/96) qui recommande de limiter la quantité d'azote susceptible d'être épandue annuellement sous forme de lisier à $170 \mathrm{~kg} \mathrm{~N} \mathrm{ha}^{-1}$ (en valeur moyenne sur chaque exploitation). Les teneurs moyennes de N, P et $\mathrm{K}$ dans le lisier sont respectivement $0,5,0,17$ et $0,25 \%$ (Coppenet, 1974). Un apport de $170 \mathrm{~kg} \mathrm{~N}$ $\mathrm{ha}^{-1}$ se traduisait jusqu'alors par des apports moyens de $60 \mathrm{~kg} \mathrm{Pha}^{-1}$ et de $83 \mathrm{~kg} \mathrm{Kha}^{-1}$. Ces quantités, en particulier pour le phosphore (Boniface et Trocmé, 1988 ; Coppenet, 1988), sont significativement supérieures à celles exportées par les cultures qui reçoivent de tels épandages. La conséquence directe en fut, dans de nombreuses parcelles, un accroissement des teneurs du phosphore extrait du sol par la méthode Dyer (Coppenet et al, 1993). Des contraintes topographiques ont été ajoutées à la contrainte azote : elles limitent les surfaces susceptibles de recevoir du lisier. Le législateur exige des éle- veurs qu'ils présentent un plan d'épandage du lisier avant d'autoriser toute création, ou agrandissement, d'une porcherie «industrielle». Cet impératif aboutit, en particulier dans certaines régions bretonnes, à supprimer toute possibilité de création ou d'agrandissement de porcheries.

Pour pouvoir respecter la législation sans pénaliser les producteurs de porcs, au moins huit procédés industriels permettant d'éliminer, par dénitrification après nitrification, le maximum possible d'azote présent dans le lisier ont été mis au point en France (brevet $n^{\circ} 9214003$; Martinez et Guiziou, 1995). Dans la plupart des procédés le produit final ne contient plus que 20 à $25 \%$ de l'azote initial, mais sa teneur en $\mathrm{P}$ et de $\mathrm{K}$ est automatiquement multipliée par 4 ou 5 . Les autorisations d'épandages de lisiers ont été établies en ne considérant que l'azote. En conséquence, les opérations de nitrification-dénitrification du lisier ont pour résultats arithmétiques des épandages sur les sols de quantités de phosphore et de potassium se situant de 350 à $850 \mathrm{~kg} \mathrm{ha}^{-1}$. Les risques de transfert du phosphore vers les eaux, puis de leur eutrophisation (Hénin, 1990 ; Sharpley et Withers, 1994), sont actuellement avancés pour envisager d'interdire, également par voie législative, des épandages de phosphore aussi excédentaires par rapport aux prélèvements des cultures lorsque le phosphore est apporté par un lisier, ou par un produit dérivé par dénitrification. Mais dans le même temps rien n'empêche légalement un agriculteur d'apporter sur ses parcelles du phosphore et du potassium, mais aussi de l'azote, à des doses aussi élevées s'il utilise des engrais minéraux inclus dans la norme NF 42001.

L'effet, sur les propriétés du sol, de doses aussi importantes, le plus souvent cumulatives, surtout en présence de composés organiques (N'dayegamiye et Cote, 1989 ; Oberson, 1993 ; Oberson et al, 1993), n'a pas fait l'objet d'études systématiques (Boniface et Trocmé, 1988 ; Bosc, 1988 ; Tran et N'dayegamiye, 1995), même si des observations ont déjà été réalisées en Bretagne (Coppenet, 1974 ; 1985 ; Duval, 1988 ; 
Coppenet et al, 1993). C'est afin de combler en partie ce manque que cette étude a été entreprise, tout particulièrement pour préciser le devenir du phosphore dans ces conditions.

Pour ce faire, il fallait avoir accès à un dispositif expérimental de plein champ recevant des apports répétés et connus de lisiers. Puis il fallait caractériser le phosphore biodisponible des sols, puisque seule cette forme conduit à l'eutrophisation des eaux de surface (Sharpley et Withers, 1994). Le dispositif Solépur, mis en place en Bretagne par le Cemagref, permettait de satisfaire à la première condition ; la méthode des cinétiques d'échanges isotopiques des ions phosphate dans des sytèmes sol-solution à la seconde.

\section{MATÉRIEL ET MÉTHODES}

\section{Le modèle expérimental Solépur}

\section{Le dispositif expérimental}

Il a été mis en place en 1990 par le Cemagref en Bretagne à Plouvorn (Finistère). II est destiné à tester en grandeur réelle un procédé "naturel" de nitrification-dénitrification de tout ou partie de l'azote contenu dans les lisiers. Le système complet a été décrit en détail (Martinez, 1994 ; Martinez et Guiziou, 1995). II s'agit d'une parcelle de $3280 \mathrm{~m}^{2}$ aménagée en drainage intégral, grâce à un film étanche de polyane de $15 / 100$ situé à $80 \mathrm{~cm}$ de profondeur. Le dispositif a d'abord été creusé couche par couche puis, et après pose du film étanche, des drains et de cailloutis permettant un drainage sans colmatage, les couches $40-60 \mathrm{~cm}$, puis $20-40 \mathrm{~cm}$, puis $0-20 \mathrm{~cm}$ ont été successivement remises en place. Le dispositif comporte une parcelle témoin, $T$, qui ne reçoit pas de lisier. La parcelle qui reçoit des épandages massifs et répétés de lisiers de porc, nommée parcelle épuratrice
Solépur, a été divisée, à des fins d'analyse statistique, en quatre sous parcelles A, B, C et D. Toutes ces parcelles ont été cultivées avec du Lolium perenne. Le Lolium est renouvelé chaque année au moyen d'un semis avec façons culturales jusqu'à $20 \mathrm{~cm}$ de profondeur. Le Lolium est régulièrement récolté puis analysé. L'ammonification de l'azote du lisier puis la nitrification ont lieu dans le sol ; les nitrates collectés dans les solutions drainantes sont dénitrifiés dans un réacteur de dénitrification ouvert séparé.

\section{Le sol}

II s'agit d'un sol brun faiblement lessivé à texture limon moyen sableux. Ses principales caractéristiques, au moment de l'implantation du dispositif, sont résumées dans le tableau I.

\section{Le lisier appliqué}

Les apports, exprimés en $\mathrm{m}^{3} \mathrm{ha}^{-1}$, ont été les suivants au cours des années : $1991: 1436,1992: 793,1993$ : 964, 1994 : 726 soit un total de $3919 \mathrm{~m}^{3} \mathrm{ha}^{-1}$. Ces apports sont fractionnés en six fois de fin mars à fin octobre. En 1994, les apports ont eu lieu les 10/05, 08/6, 24/06 et 20/07.

La composition des lisiers est extrêmement variable (Coppenet, 1974 ; Bril et Salomons, 1990 ; Japenga et Harmsen, 1990 ; Bernal et al, 1993a). Cette observation a été confirmée par nos mesures. Ainsi, en 1994, la teneur du phosphore a varié de 0,15 à $0,30 \%$. Les valeurs moyennes, exprimées en $\mathrm{mg}$ d'élément par $\mathrm{g}$ de lisier, des teneurs à prendre en compte pour établir des bilans matière dans le dispositif sont les suivantes: $\mathrm{N}=4,8, \mathrm{P}=1,75, \mathrm{~K}=2,9, \mathrm{Ca}=2,5$ et $\mathrm{Mg}=$ 0,55 . L'apport d'azote, cumulé pour les 4 années, a été de 18,9 t N ha-1 (Devloo, 1994). Les apports de phosphore, établis sur la base des analyses de lisiers, ont été de : $1412 \mathrm{~kg} \mathrm{P}^{-1}$ en 1991, $1233 \mathrm{~kg} \mathrm{P}^{-1}$ en

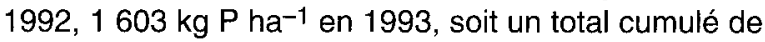
$4248 \mathrm{~kg} \mathrm{P} \mathrm{ha}^{-1}$ jusqu'au prélèvement de mars 1994. En 1994, l'apport a été de $1616 \mathrm{~kg} \mathrm{P} \mathrm{ha}^{-1}$.

Tableau I. Principales caractéristiques physicochimiques, en début d'expérimentation, de la terre des trois horizons du dispositif Solépur.

Granulométrie $(\mu \mathrm{m})$

\begin{tabular}{|c|c|c|c|c|c|c|c|c|}
\hline $\begin{array}{l}0-2 \\
(\%)\end{array}$ & $\begin{array}{c}2-20 \\
(\%)\end{array}$ & $\begin{array}{c}20-50 \\
(\%)\end{array}$ & $\begin{array}{c}50-200 \\
(\%)\end{array}$ & $\begin{array}{c}200-2000 \\
(\%)\end{array}$ & $p H$ & $\begin{array}{c}C \\
(\%)\end{array}$ & $\begin{array}{c}N \\
(\%)\end{array}$ & $\begin{array}{c}C E C \\
\left(\mathrm{cmol}^{+} \mathrm{kg}^{-1}\right)\end{array}$ \\
\hline 14,1 & 22,4 & 41,0 & 17,1 & 5,4 & 5,9 & 1,82 & 0,176 & 8,4 \\
\hline 13,2 & 21,9 & 37,5 & 20,0 & 7,4 & 5,2 & 1,38 & 0,139 & 7,9 \\
\hline 17,5 & 17,1 & 33,6 & 22,3 & 9,5 & 5,1 & 0,71 & 0,071 & 7,5 \\
\hline
\end{tabular}




\section{Échantillons de terre prélevés et analysés}

Chaque année, au mois de mars juste avant le premier apport de lisier, des échantillons de terre ont été prélevés. Ils ont été réalisés comme suit : dans chaque parcelle $T, A, B, C$ et $D$, neuf prélèvements sont effectués à l'aide d'une tarière manuelle. Les horizons $0-20$, 20-40 et $40-60 \mathrm{~cm}$ sont séparés. Les échantillons sont mélangés, ce qui représente une masse de sol de 12 à $15 \mathrm{~kg}$ par parcelle et par horizon. L'ensemble est tamisé sur place à $5 \mathrm{~mm}$ et un échantillon moyen de 1 à $2 \mathrm{~kg}$ est réalisé puis mis en glacière pour être conservé.

De plus, en 1994, des échantillons de terre ont été prélevés dans l'horizon $0-30 \mathrm{~cm}$ avant chaque apport soit les 19/04, 31/05, 15/06 et 01/07.

Des analyses de phosphore biodisponible, du $\mathrm{pH}$ et des cations de la solution du sol ont été effectuées pour l'ensemble des échantillons prélevés en 1991, 1992,1993 et 1994. Des analyses de phosphore total n'ont été effectuées que sur les échantillons prélevés en 1991 et 1994.

\section{Caractérisation du phosphore biodisponible}

En analyse de routine le phosphore supposé "assimilable" est extrait du sol soit par des méthodes chimiques, unique ou séquentielles, soit par des méthodes physiques (Judel et al, 1985). Ces pratiques donnent accès à une quantité, celle qui est extraite, sans jugement de valeur sur sa qualité nutritive à l'égard des plantes (Juste, 1988). Mais «l'analyse du sol, même en y ajoutant celle du sous-sol, ne peut fournir en aucun cas une mesure absolue des masses de $P$ réellement disponibles, mais seulement le sens de variation du statut phosphaté du sol en fonction du bilan cultural» (Boniface et Trocmé, 1988). Une caractérisation plus élaborée et plus explicative du devenir du phosphore disponible pour les plantes, c'est-à-dire de la forme du phosphore du sol susceptible d'être prélevé par les cultures, nécessite de déterminer trois paramètres expérimentaux susceptibles de décrire les trois facteurs suivants : intensité, quantité et capacité, ce dernier devant rendre compte de l'aptitude du sol à maintenir le facteur quantité constant lorsque le facteur intensité varie du fait soit d'un apport soit d'un prélèvement (Beckett et White, 1964 ; Gachon, 1977).

Les plantes ne prélèvent le phosphore dont elles ont besoin que dans le pool des ions phosphate du sol susceptibles de participer aux échanges isotopiques (Frossard et al, 1994 ; Morel et Plenchette, 1994) : ce pool, et lui seul, correspond donc au phosphore biodisponible. C'est pourquoi, en l'état actuel des connaissances et des méthodes existantes, seule la technique des cinétiques d'échanges isotopiques des ions phosphate dans des systèmes sol-solution en état stationnaire permet d'accéder, sur un échantillon unique et au cours d'une seule expérience, à des paramètres expérimentaux représentatifs des facteurs intensité, quantité et capacité (Fardeau, 1993). C'est pourquoi ce phosphore est ici nommé phosphore biodisponible.

\section{Extraction chimique : la méthode Dyer avec utilisation de l'acide citrique}

Dans ce sol de Bretagne initialement acide (tableau I), les analyses de routine ont été réalisées avec la méthode de Dyer, quoiqu'il eût été possible d'utiliser la méthode Joret-Hébert ou la méthode Olsen puisque les résultats obtenus avec les trois méthodes sont, dans les conditions bretonnes et en présence d'apports de lisiers, hautement corrélées (Duval, 1988). Elle consiste désormais à extraire le phosphore avec de l'acide citrique à $2 \%$, dans un mélange sol-extractant de rapport $1 / 5$, durant 16 heures.

Ce type d'extraction a également été pratiqué sur de l'inositol hexaphosphate de calcium et magnésium (IHP-Ca, Mg), principale forme de phosphore présente dans les lisiers de porcs (Cromwell, 1992). Un g d'IHP. $\mathrm{Ca}, \mathrm{Mg}$, à $18,6 \%$ de phosphore total, a été agité en présence de $100 \mathrm{~mL}$ d'acide citrique à $2 \%$ selon la méthode d'extraction Dyer. Les ions phosphate libérés ont été dosés, à l'issue de l'extraction, par colorimétrie à froid pour éviter toute hydrolyse en milieu acide et à chaud de I'IHP-Ca, Mg.

\section{La technique des échanges isotopiques des ions phosphate dans les systèmes sol-solution de sol}

Elle a été décrite en détail antérieurement (Fardeau et al, 1988 ; Fardeau, 1993). Les cinétiques d'échanges sont réalisées dans des systèmes sol-solution de sol de rapport 1/10 ayant atteint un état stationnaire obtenu par une agitation d'une nuit. Dans ce mélange sol-terre agité alors par un barreau magnétique, on injecte à un temps $t=0$ une quantité $\mathrm{R}$ de radioactivité sous forme d'ions phosphate sans entraîneur et marqués au $32 \mathrm{P}$; puis on effectue, à des instants $t_{1}, t_{2}, t_{3}$, $\ldots, t_{n}$, des prélèvements dans le mélange que l'on filtre immédiatement sur filtres à pores de $0,025 \mu \mathrm{m}$. La radioactivité restant en solution à chaque instant de prélèvement est déterminée par comptage en scintillation liquide, de préférence à un comptage par effet Cerenkov qui ne permet pas les corrections de «quenching". La concentration du phosphore, sous forme d'ions phosphate, dans la solution du sol est déterminée désormais par une technique colorimétrique utilisant le vert malachite (Van Veldhoven et Mannaerts, 1987 ; Novozamski et al, 1993). Mais le polyvinylalcool (PVA) a été modifié : il s'agit d'un PVA de poids moléculaire 72000 (Référence Merck : 821038). Cette technique est plus sensible que toutes les méthodes antérieures utilisant une réduction du phosphomolybdate et est insensible à la silice.

Les données obtenues permettent de disposer des trois paramètres représentatifs des facteurs intensité, quantité et capacité. La concentration, $\mathrm{C}_{\mathrm{p}}$, illustre le facteur intensité (Barber, 1984). La quantité $E_{\text {Pie1 }}=10$ $C_{P}\left(R / r_{1}\right)$ de phosphore échangée durant la première minute d'échange isotopique est retenue comme paramètre représentatif du facteur quantité. Le rapport $\mathrm{E}_{\mathrm{Pie} 1} / \mathrm{C}_{\mathrm{P}}$ est par définition le facteur capacité ou pouvoir tampon instantané du sol pour les ions phosphate (Fardeau, 1993). II a pour valeur numérique $10 \mathrm{R} / \mathrm{r}_{1}$, soit 10 fois le rapport de la radioactivité introduite, $R$, à 
la radioactivité, $r_{1}$, restant en solution après 1 minute d'échange. Une troisième donnée, $n$, est également déduite de la cinétique des échanges isotopiques, c'est-à-dire du transfert, en fonction du temps, des ions phosphate de la phase liquide vers la phase solide (Fardeau et al, 1991). Cette donnée $n$ permet de décrire les transferts potentiels d'ions phosphate vers la solution du sol en fonction du temps, en particulier lorsque le phosphore présent dans la solution de sol est prélevé par les racines ; il s'agit d'un pouvoir tampon différé. Elle permet également, par analyse compartimentale, le calcul de la taille de compartiments de phosphore présent dans le sol et dont les ions sont susceptibles d'atteindre plus ou moins rapidement la solution du sol (Fardeau, 1993).

\section{Analyse des cations présents dans des solutions du sol}

Les teneurs des cations ont été déterminées par absorption atomique d'une part dans la solution du sol extraite par filtration sur filtres millipores à pores de $0,025 \mu \mathrm{m}$ utilisée pour déterminer la concentration, $C_{p}$, des ions phosphate et d'autre part dans les eaux de drainage issues des parcelles recevant le lisier.

Les pH ont été mesurés dans les mélanges sol-solution de sol, de rapport $1 / 10$, utilisés pour les déterminations du phosphore biodisponible par la technique des cinétiques d'échanges isotopiques.

\section{Démarche statistique}

En raison des quantités très élevées des apports, des approches statistiques peu élaborées, tel le calcul des écart types, ont été suffisantes pour apprécier des différences significatives.

De plus les variations sur des données expérimentales telles que celles issues des cinétiques d'échanges isotopiques sont désormais très bien établies. Le coefficient de variation qui était de $10 \%$ sur les valeurs de $\mathrm{C}_{\mathrm{P}}$ est désormais de $5 \%$ avec l'utilisation de la méthode au vert malachite, de $4 \%$ sur les valeurs de $r_{1} / R$ et de $3 \%$ sur les valeurs de $E_{\text {Pie1 }}$ (Fardeau, 1993). Ils sont plus faibles pour $E_{\text {pie1 }}$ que pour les variables $C_{P}$ et $r_{1} / R$ qui sont utilisées pour calculer $E_{\text {Pie1 }}$ car $E_{\text {Pie1 }}$ est en fait l'inverse d'une teneur isotopique pour lesquelles les variations sont, quel que soit l'élément, toujours inférieures aux variations de chacun des isotopes constituants de la composition isotopique de l'élément analysé.

\section{RÉSULTATS ET DISCUSSIONS}

\section{Observations générales sur le dispositif}

La teneur de l'azote total croît essentiellement dans l'horizon de surface avec les apports de lisiers ; elle était en 1993 de $0,20 \%$ dans la parcelle témoin et de $0,25 \% \pm 0,011 \%$ dans les parcelles recevant le lisier. Celle du carbone évolue de manière similaire : elle est de 1,95\% sans apport et atteint $2,54 \% \pm 0,19 \%$ avec apport. Ce résultat illustre que, dans ce dispositif expérimental mis en place pour transformer l'azote organique du lisier en azote moléculaire, environ $20 \%$ de l'azote apporté a été insensible, à l'échelle du temps d'observation, aux processus de minéralisation. Pour le carbone cette valeur est d'environ $25 \%$. Ces valeurs sont inférieures à celles observées dans d'autres dispositifs : $40 \%$ (Bernal et al, 1993b) ou $50 \%$ (Whitehead et al, 1989). Les différences de minéralisation du carbone ont très probablement pour origine les conditions de circulation de l'oxygène dans les différents dispositifs. II faut signaler qu'au cours des 4 années d'apport la couleur du sol est devenue plus sombre. Selon les couleurs «Munsell», elle est 10 YR 5,5/4 dans la parcelle témoin et 10 YR 4,5/3 dans les parcelles Solépur traitées avec le lisier. De semblables observations, toujours expliquées par la restitution de déjections animales pratiquées sur un espace de temps infiniment plus grand - puisqu'il s'agit de quelques siècles (Pape, 1970) -, ont très probablement pour origine l'accumulation de sels biliaires, toujours colorés, et particulièrement peu dégradables.

\section{Cinétique pluriannuelle}

\section{Phosphore biodisponible}

\section{Extraction Dyer}

Les valeurs moyennes obtenues à partir des échantillons des quatre sous-parcelles $A, B, C$ et D Solépur et celle de la parcelle témoin $T$ sont regroupées dans le tableau II. La quantité de phosphore extraite par le réactif Dyer croît au cours des ans avec les quantités de lisier apportées. Ces résultats confirment les observations obtenues antérieurement en suivant, au cours des ans, des parcelles "agriculteur" recevant régulièrement du lisier (Coppenet, 1974, 1985, 1988 ; Coppenet et al, 1993). Mais ils montrent aussi que du phosphore a significativement migré en profondeur à l'issue de ces apports de lisiers.

\section{Phosphore isotopiquement échangeable}

Les résultats ont été regroupés dans les tableaux III, IV et $\mathrm{V}$ pour les facteurs quantité, $\mathrm{E}_{\mathrm{Pie} 1}$, inten- 
Tableau II. Quantités de phosphore, exprimées en mgP ( $\mathrm{kg} \mathrm{sol}^{-1}$, extraites par la méthode Dyer dans la parcelle témoin, $T$, et moyenne des quatre sous-parcelles Solépur, $A, B, C, D$ (entre parenthèses : écart type).

\begin{tabular}{|c|c|c|c|c|c|c|c|c|c|}
\hline & & 991 & & 992 & & & 1993 & & 1994 \\
\hline & $T$ & Solépur & $T$ & Solépur & & $T$ & Solépur & $T$ & Solépur \\
\hline $0-20 \mathrm{~cm}$ & 104 & $158(3)$ & 96 & $455(19)$ & & 68 & $901(10)$ & 100 & $1115(25)$ \\
\hline $20-40 \mathrm{~cm}$ & 55 & $96(2)$ & 41 & $179(5,7)$ & & 74 & $242(79)$ & 55 & $513(24)$ \\
\hline $40-60 \mathrm{~cm}$ & 6,5 & $22(6,5)$ & 4,3 & $26(1,3)$ & & 8,7 & $51(4)$ & 4,3 & $42(2,6)$ \\
\hline
\end{tabular}

sité, $\mathrm{CP}$, et capacité, ou pouvoir tampon instantané, $10 \mathrm{R} / \mathrm{r}_{1}$.

Les quantités $E_{P i e 1}$ de la parcelle $T$ ne recevant pas de lisier doivent être considérées comme invariantes au cours des années successives. Les valeurs observées en 1993 pour la parcelle $T$ dans les horizons $0-20 \mathrm{~cm}$ et 20-40 cm illustrent un coup de tarière un peu profond. En revanche les quantités $E_{\text {Pie } 1}$ du dispositif Solépur croissent au cours des ans dans les trois horizons. Elles témoignent donc, comme celles obtenues avec la méthode Dyer, d'une migration du phosphore.

Or le phosphore présent dans des déjections porcines n'a pour origine que le phosphore préalablement contenu dans les aliments du bétail. Cette origine explique pourquoi ce phosphore est à $80 \%$ en moyenne à l'instant de son rejet dans le milieu environnant inclus dans des phytates de calcium et magnésium ou IHP-Ca, Mg (Cromwell, 1992 ; Zhang et al, 1993). Or les ions phosphate de I'IHP-Na, comme ceux de l'IHP-Ca, Mg ou de I'IHP-Fe ne subissent pas d'échanges isotopiques (Fardeau et al, 1968). L'accroissement, après l'application du lisier, des teneurs du phosphore instantanément isotopiquement échan- geable, $E_{\text {Pie1 }}$, dans les horizons de surface et la migration d'une partie du phosphore ne peuvent donc être expliqués que par une libération d'ions phosphate résultant de l'hydrolyse de l'IHP-Ca, $\mathrm{Mg}$ par des phytases présentes dans le sol (Bowman et Cole, 1988a, b ; Kucey et al, 1989 ; Mousain, 1989).

La régression linéaire entre les couples $P_{\text {Dyer }}$ - E Eie1 pour chaque prélèvement est représentée par :

$$
P_{\text {Dyer }}=21,65 \mathrm{E}_{\text {Pie } 1}+12,76
$$

avec $r^{2}=0,87$ pour $n=60$

Le coefficient de détermination, $r^{2}$, est élevé. Mais l'examen visuel des données individuelles placées sur un graphique fait apparaître que le rapport $P_{\text {Dyer }} / E_{\text {Pie } 1}$ est en moyenne de 10 pour les échantillons prélevés entre 40 et $60 \mathrm{~cm}$ de profondeur qui sont, de fait, ceux pour lesquels les teneurs sont les plus faibles, et qu'il est en moyenne de 26 pour les échantillons de surface et ceux de l'horizon $20-40 \mathrm{~cm}$. Pour un horizon donné d'une parcelle donnée, le rapport $P_{\text {Dyer }} / E_{\text {Pie } 1}$ varie également au cours des

Tableau III. Évolution des quantités $E_{\text {Pie1 }}$, exprimées en mgP (kg sol)-1, de phosphore isotopiquement échangé en 1 minute en fonction des traitements et du temps.

\begin{tabular}{|c|c|c|c|c|c|c|c|c|}
\hline & \multicolumn{2}{|c|}{1991} & \multicolumn{2}{|c|}{1992} & \multicolumn{2}{|c|}{1993} & \multicolumn{2}{|c|}{1994} \\
\hline & $T$ & Solépur & $T$ & Solépur & $T$ & Solépur & $T$ & Solépur \\
\hline$-20 \mathrm{~cm}$ & 4,5 & 5,6 & 3,7 & 24,9 & 3,9 & 32,4 & 5,5 & 53,8 \\
\hline $0-40 \mathrm{~cm}$ & 2,0 & 3,8 & 3,8 & 11,2 & 2,5 & 7,6 & 1,7 & 22,2 \\
\hline $0-60 \mathrm{~cm}$ & 1,5 & 1,8 & 0,36 & 1,3 & 1,1 & 5,4 & 0,56 & 4,0 \\
\hline
\end{tabular}


Tableau IV. Évolution des concentrations de phosphore, exprimées en $\mathrm{mgP} \mathrm{L}^{-1}$, en fonction des traitements et du temps.

\begin{tabular}{|c|c|c|c|c|c|c|c|c|}
\hline & \multicolumn{2}{|c|}{1991} & \multicolumn{2}{|c|}{1992} & \multicolumn{2}{|c|}{1993} & \multicolumn{2}{|c|}{1994} \\
\hline & $T$ & Solépur & $T$ & Solépur & $T$ & Solépur & $T$ & Solépur \\
\hline $0-20 \mathrm{~cm}$ & 0,053 & 0,062 & 0,043 & 0,53 & 0,035 & 0,95 & 0,05 & 1,87 \\
\hline $20-40 \mathrm{~cm}$ & 0,010 & 0,022 & 0,0068 & 0,15 & 0,015 & 0,11 & 0,01 & 0,35 \\
\hline $40-60 \mathrm{~cm}$ & 0,0013 & 0,0026 & 0,0003 & 0,0023 & 0,002 & 0,023 & 0,0014 & 0,014 \\
\hline
\end{tabular}

années, c'est-à-dire avec la charge appliquée et le temps écoulé après l'apport. Ces observations illustrent d'abord, s'il en était encore nécessaire, qu'il est illusoire, à l'issue d'une extraction chimique, d'utiliser un facteur constant pour espérer connaître la quantité de phosphore réellement assimilable (Nirel et Morel, 1990).

Les variations du rapport $P_{\text {Dyer }} / E_{\mathrm{Pie} 1}$ ont pour origine le comportement différent de I'IHP-Ca, $\mathrm{Mg}$ vis-à-vis de l'acide citrique et de l'échange isotopique. La quantité de phosphore extrait de de I'IHP-Ca,Mg par la méthode Dyer, puis dosé, correspond à la totalité du phosphore contenu dans I'IHP-Ca,Mg. Or le phosphore présent dans I'IHP-Ca, Mg n'est pas dosable sans hydrolyse préalable. On est donc en droit de conclure que l'acide citrique à $2 \%$ hydrolyse I'IHP-Ca,Mg. Ainsi en utilisant la méthode d'extraction de Dyer, le phosphore contenu dans lisier est considéré comme assimilable dès qu'il est appliqué au sol. A l'inverse, l'utilisation de la technique des échanges isotopiques appliquée à de I'IHP. $\mathrm{Ca}, \mathrm{Mg}$ permet de démontrer que les phosphates liés à l'inositol ne sont pas isotopiquement échangeables (Fardeau et al, 1968) ; en conséquence, les ions phosphate de I'IHP-Ca,Mg ne peuvent pas, en l'état, être considérés comme biodisponibles. Ce faisceau de résultats permet d'expliquer les différences d'appréciation du phosphore assimilable observées entre la méthode d'extraction chimique à l'acide citrique et la méthode d'échanges isotopiques. La méthode d'extraction à l'acide citrique surestime les quantités de phosphore biodisponible dans un sol ayant reçu très récemment du lisier. Des conclusions similaires ont été faites au sujet d'autres extractions acides (Robinson et al, 1995 ; Sharpley et Smith, 1995).

Le rapport $\mathrm{P}$-Dyer/ $\mathrm{E}_{\mathrm{Pie} 1}$ devrait donc croître avec les quantités de lisier appliquées: mais tel n'est pas le cas. En fait, après épandage sur un sol non calcaire, deux mécanismes antagonistes ont lieu : d'une part, une partie de l'IHP-Ca,Mg est hydrolysée par des phytases microbiennes du sol (Dighton, 1991) et libère ses ions phosphate qui, pour une fraction, accroissent la taille du pool biodisponible ; d'autre part, l'autre partie de I'IHP-Ca,Mg est transformée en IHP-Fe non hydrolysable par la majorité des phytases (Cosgrove, 1980). La conséquence en est une évolution très complexe du rapport $P$-Dyer/ $E_{\text {Pie1 }}$ dans un sol non saturé en calcium. L'évolution

Tableau V. Évolution du facteur capacité, $E_{\mathrm{Pie} 1} / C_{\mathrm{P}}$, exprimé en $L(\mathrm{~kg} \mathrm{sol})^{-1}$, en fonction des traitements et du temps.

\begin{tabular}{|c|c|c|c|c|c|c|c|c|}
\hline & \multicolumn{2}{|c|}{1991} & \multicolumn{2}{|c|}{1992} & \multicolumn{2}{|c|}{1993} & \multicolumn{2}{|c|}{1994} \\
\hline & $T$ & Solépur & $T$ & Solépur & $T$ & Solépur & $T$ & Solépur \\
\hline $0-20 \mathrm{~cm}$ & 85 & 90 & 86 & 48 & 111 & 34 & 109 & 29 \\
\hline $20-40 \mathrm{~cm}$ & 200 & 172 & 235 & 77 & 166 & 67 & 170 & 63 \\
\hline $40-60 \mathrm{~cm}$ & 1150 & 692 & 1200 & 550 & 550 & 232 & 400 & 285 \\
\hline
\end{tabular}


du lisier devrait donc être sensiblement différente dans les sols calcaires : la totalité du phosphore du phytate appliqué au sol doit être à terme hydrolysée (Bernal et al, 1993b ; Papini et al, 1994). En raison des quantités massives de lisiers appliqués dans le dispositif Solépur, et dans de nombreuses parcelles d'agriculteurs (Coppenet et al, 1993), le pH du sol croît légèrement au fil des ans, ce qui limite la transformation de I'IHP-Ca,Mg en IHP-Fe non hydrolysable. Les concentrations, $\mathrm{CP}$, des ions phosphate (tableau IV) illustrent que, dans ce type de sol et aux doses d'apport utilisées, la concentration de $0,2 \mathrm{mgP} \mathrm{L}^{-1}$ à partir de laquelle du phosphore commence à migrer en quantité significative dans la couche sous jacente à celle où il a été placé a été atteinte dans l'horizon de surface de ce dispositif dès la seconde année des apports malgré l'existence d'un pouvoir fixateur initialement très élevé.

Les apports de lisiers entraînent une diminution significative du facteur capacité (tableau V), et par voie de conséquence du pouvoir fixateur pour les ions phosphate dans les trois horizons. Trois mécanismes concourent à cette diminution : i) tout apport, dans un système terre-eau, de phosphore isotopiquement échangeable entraîne une diminution du facteur capacité (Hartikenen, 1991), ou du rapport $E_{P i e 1} / C_{P}$ (Fardeau, 1993) ; l'importance de cette diminution dépend de la nature des constituants du sol ; ii) de nombreux composés organiques diminuent le facteur capacité (Frossard, 1985) ; iii) le pouvoir fixateur des sols diminue le plus souvent lorsque le $\mathrm{pH}$ du sol passe de l'acidité à la neutralité (Barbier et al, 1965). Cependant, dans le cas de podzols, des situations inverses semblent avoir été observées au Canada. Nos données ne sont pas suffisantes pour faire la part de chacun de ces mécanismes dans la diminution du facteur capacité, ni même de les hiérarchiser.
Analyse, en termes de bilan, du devenir du phosphore appliqué sous forme de lisier

La détermination du phosphore total de quelques échantillons de terre permet d'apprécier i) la qualité du bilan entrée ; ii) l'importance des transferts de phosphore en profondeur ; iii) le taux de recouvrement sous forme extractible du phosphore appliqué (tableau VI).

En tenant compte des densités des différents horizons, l'accroissement de $\mathrm{P}$ total dans la première couche correspondrait à un apport de $2366 \mathrm{~kg} \mathrm{P} \mathrm{ha}^{-1}$, celui de la seconde à $1058 \mathrm{kgP}$ $\mathrm{ha}^{-1}$ et celui de la troisième à $300 \mathrm{kgP}$ ha-1, soit un total de $3724 \mathrm{kgP}$ ha ${ }^{-1}$. L'apport, calculé à partir des teneurs mesurées dans les lisiers est de $4248 \mathrm{kgP} \mathrm{ha}^{-1}$, soit un taux de recouvrement de $3724 / 4248$ soit $87,7 \%$. Il est bon de garder en mémoire que la détermination du $P$ total dans des échantillons de terre est connue à $100 \mathrm{mgP}$ $(\mathrm{kg})^{-1}$ près, soit environ $300 \mathrm{~kg} \mathrm{P}^{-1}$ (Boniface et Trocmé, 1968). Si, à la quantité calculée à partir de l'analyse de sol, on ajoute celle cumulée exportée avec les récoltes de ray-grass, à savoir pour les 4 années $150 \mathrm{~kg} \mathrm{P}^{-1}$, soit $3,5 \%$ de l'apport, la valeur finale du bilan calculé est de $3874 \mathrm{kgP} \mathrm{ha}^{-1}$. Elle est bien du même ordre de grandeur que celle appréciée par les apports de lisiers. La quantité de phosphore ayant migré audelà de $20 \mathrm{~cm}$ serait donc de $1358 \mathrm{~kg} \mathrm{P} \mathrm{ha}^{-1}$ soit $32 \%$ de l'apport.

L'apport de $2366 \mathrm{kgP} \mathrm{ha}^{-1}$ dans l'horizon $0-20 \mathrm{~cm}$ a accru la quantité extraite par la méthode Dyer de $711 \mathrm{mgP} \mathrm{kg}^{-1}$, soit $300 \mathrm{mgP}$ $\mathrm{kg}^{-1}$ pour $1000 \mathrm{kgP}^{-1}$ appliqué. Cet accroissement de la quantité Dyer est trois fois plus élevée que celle récemment proposée (Boniface et Trocmé, 1988), et observée à l'issue d'apports de phosphore effectués uniquement sous forme minérale. Certes une erreur d'appréciation doit être imputée à l'extraction des phytates non encore hydrolysés ; mais la même conclusion est

Tableau VI. Bilan du phosphore dans la sous-parcelle A ayant reçu du lisier. Les quantités sont exprimées en mgP (kg sol) ${ }^{-1}$.

\begin{tabular}{|c|c|c|c|c|c|c|c|c|c|}
\hline & \multicolumn{2}{|c|}{$P_{\text {total }}$} & \multirow[t]{2}{*}{$\Delta P_{\text {total }}$} & \multicolumn{2}{|c|}{$P_{\text {Dyer }}$} & \multirow[t]{2}{*}{$\Delta P_{\text {Dyer }}$} & \multicolumn{2}{|c|}{$E_{\text {Pie1 }}$} & \multirow[t]{2}{*}{$\Delta E_{\text {Pie } 1}$} \\
\hline & 1991 & 1994 & & 1991 & 1994 & & 1991 & 1994 & \\
\hline $0-20 \mathrm{~cm}$ & 815 & 1725 & 910 & 190 & 901 & 711 & 7,2 & 61,8 & 54,6 \\
\hline $20-40 \mathrm{~cm}$ & 772 & 1150 & 378 & 122 & 425 & 303 & 4,6 & 30,8 & 26,2 \\
\hline $40-60 \mathrm{~cm}$ & 640 & 740 & 100 & 29 & 77 & 48 & 1,6 & 6,2 & 4,6 \\
\hline
\end{tabular}


Tableau VII. Pourcentages, par rapport au phosphore total, des quantités de phosphore extrait par la méthode de Dyer et des quantités instantanément échangeables $\mathrm{E}_{\text {Pie1 }}$.

\begin{tabular}{|c|c|c|c|c|c|c|}
\hline & \multicolumn{2}{|c|}{$P_{\text {Dyer }} / P_{\text {total }}$} & \multirow[t]{2}{*}{$\Delta P_{\text {Dyer }} / \Delta P_{\text {total }}$} & \multicolumn{2}{|c|}{$E_{\text {Piet }} / P_{\text {total }}$} & \multirow[t]{2}{*}{$\Delta E_{P i e 1} / \Delta P_{\text {total }}$} \\
\hline & 1991 & 1994 & & 1991 & 1994 & \\
\hline $0-20 \mathrm{~cm}$ & 23,4 & 52,2 & 78 & 0,88 & 3,6 & 6,0 \\
\hline $20-40 \mathrm{~cm}$ & 15,8 & 37,0 & 80 & 0,80 & 2,7 & 6,9 \\
\hline $40-60 \mathrm{~cm}$ & 4,4 & 10 & 48 & 0,24 & 0,84 & 4,6 \\
\hline
\end{tabular}

applicable au phosphore instantanément échangeable, $E_{\mathrm{Pie} 1}$, la fraction du supplément de $P$ dans l'horizon restant échangeable après ces apports étant deux à trois fois supérieure à celle observée en moyenne avec des apports minéraux dans des sols dont le pouvoir fixateur est généralement moins élevé que celui des parcelles Solépur (Fardeau, 1993). On est donc en droit de conclure que, dans le cas des apports de lisiers, la présence de matières organiques associées au phosphore contribue au maintien de la mobilité du phosphore dans les profils, la matière organique limitant les réactions de cristallisation des ions phosphate avec les cations du sol (Syers et Lu Ru-kun, 1990).

\section{Analyse compartimentale du phosphore isotopiquement échangeable}

Les résultats de cette analyse (tableau VIII) ont été obtenus en utilisant le paramètre $n$ caractéri- sant la vitesse de transfert des ions phosphate de la phase solide vers la phase liquide déterminée en analysant les transferts opposés d'ions phosphate marqués au ${ }^{32} \mathrm{P}$ (Fardeau, 1993). Les résultats ne concernent que les échantillons prélevés en 1994 dans la parcelle témoin et dans celles ayant reçu du lisier.

À la différence des observations faites après des apports d'engrais minéraux, ces données illustrent que le phosphore appliqué avec le lisier contribue à accroître tous les compartiments, en particulier les plus mobiles. Ce résultat confirme que des matières organiques, du type de celles présentes dans le lisier, sont des agents qui vont freiner, voire inhiber, la cristallisation des composés phosphatés dans les sols (Frossard, 1985) : elles vont, en autorisant le maintien de concentrations plus élevées qu'elles ne le seraient en leur absence, favoriser le maintien à l'état mobile des ions phosphate, donc leur possibilité de migration.

Tableau VIII. Analyse compartimentale du phosphore échangeable dans les échantillons prélevés en 1994. Compartiment $E_{\text {Pie1 }}: P$ instantanément disponible. Compartiment $C_{1}: P$ mobile entre 1 minute et 1 jour. Compartiment $\mathrm{C}_{2}: \mathrm{P}$ mobile entre 1 jour et 3 mois. Compartiment $C_{3}: P$ mobile entre 3 mois et 1 an. Compartiment $C_{4}: P$ immobile à l'échelle annuelle.

Échantillons

Compartiments

$\begin{array}{lllll}E_{\text {Pie } 1} & C_{1} & C_{2} & C_{3} & C_{4} \\ & & \left(m g P(k g \text { sol })^{-1}\right. & & \end{array}$

Témoin

$0-20 \mathrm{~cm}$

$20-40 \mathrm{~cm}$

$40-60 \mathrm{~cm}$

Solépur

$0-20 \mathrm{~cm}$

$20-40 \mathrm{~cm}$

$40-60 \mathrm{~cm}$
5,5
1,7
0,56

53,8

22,2

4,0
96

38

20

190

154

54

296
177
135


Tableau IX. Influence des apports de lisiers sur l'ambiance physicochimique dans la solution du sol analysée sur les échantillons prélevés en mars 1994 avant le premier épandage.

\begin{tabular}{|c|c|c|c|c|c|c|}
\hline \multirow{2}{*}{ Échantillons } & \multicolumn{6}{|c|}{ Éléments en solution } \\
\hline & $p H$ & $K$ & $\begin{array}{c}\mathrm{Ca} \\
\left(m g L^{-1}\right)\end{array}$ & $M g$ & $\mathrm{Na}$ & $\begin{array}{c}C E C \\
\left(\mathrm{cmol}^{+} \mathrm{kg}^{-1}\right)\end{array}$ \\
\hline \multicolumn{7}{|l|}{ Témoin } \\
\hline $0-20 \mathrm{~cm}$ & 5,7 & 4,1 & 2,5 & 0,65 & 2,8 & 8,65 \\
\hline $20-40 \mathrm{~cm}$ & 5,5 & 3,2 & 1,8 & 0,60 & 2,1 & 8,45 \\
\hline $40-60 \mathrm{~cm}$ & 4,7 & 1,8 & 1,2 & 0,52 & 2,3 & 9,35 \\
\hline \multicolumn{7}{|l|}{ Solépur } \\
\hline $0-20 \mathrm{~cm}$ & 7,3 & 40,8 & 14,5 & 5,6 & 3,4 & 12,0 \\
\hline $20-40 \mathrm{~cm}$ & 7,4 & 35,5 & 8,8 & 2,9 & 3,7 & 8,6 \\
\hline $40-60 \mathrm{~cm}$ & 6,0 & 25,7 & 2,5 & 1,5 & 4,0 & 7,0 \\
\hline
\end{tabular}

\section{Cations présents dans la solution du sol}

En présence de lisier, le $\mathrm{pH}$ a augmenté dans les trois horizons (tableau IX); ce facteur contribue, comme l'addition des matières organiques, à réduire le pouvoir fixateur du sol pour les ions phosphate. La modification la plus importante concerne la concentration du potassium dans la solution du sol. L'analyse du potassium échangeable à l'acétate d'ammonium, qui atteint désormais dans les horizons $0-20,20-40$ et $40-60 \mathrm{~cm}$ les valeurs de $2,5,2,4$ et $1,8 \mathrm{cmol}+\mathrm{kg}^{-1}$, soit 20 , 28 et $26 \%$ de la CEC, illustre que tous les horizons du profil des parcelles ayant reçu le lisier sont saturés en potassium. La conséquence en est un lessivage total du potassium qui désormais est appliqué sur la parcelle Solépur. La mesure de la concentration du potassium dans les eaux percolées, qui atteignait $70 \mathrm{mgK}^{-1}$ dès 1993, confirme cette conclusion. Les différences de concentrations du potassium observées dans les solutions de sol collectées à l'occasion de l'établissement des cinétiques d'échanges isotopiques s'expliquent par les différences de pouvoir tampon des terres à l'égard des ions potassium. L'accroissement des teneurs du magnésium confirme indirectement l'hydrolyse dans le sol de I'IHP-Ca,Mg puisque ce composé représente la source essentielle de cet élément dans ce système. L'examen des données de ce tableau illustre que les apports de lisiers n'ont pas seulement un effet sur l'azote, seul élément pris en compte dans le raisonnement légal des apports, mais sur l'ensemble des éléments susceptibles d'influencer la nutrition des plantes (Christie, 1987 ; Stevens et al, 1988), et plus généralement la qualité des sols (Cheverry, 1994).

\section{Cinétique annuelle}

Les résultats présentés ne concernent que les données issues des expériences d'échange isotopique (tableau X). L'impact des apports de lisiers sur l'ensemble des caractéristiques physicochimiques des sols est quasi instantané. La comparaison de ces données avec celles concernant les évolutions interannuelles qui portent sur des prélèvements réalisés en sortie d'hiver avant le premier apport de lisier (tableaux III, IV et IX) montre que des transformations, aboutissant à une diminution des teneurs des éléments $P, K$, $\mathrm{Ca}$ et $\mathrm{Mg}$, se déroulent pendant la période hivernale sans apport. Ces transformations sont cependant très loin d'annuler l'ensemble des modifications observées au moment des apports de lisiers.

\section{DISCUSSION : ÉPANDAGES DE LISIERS DE PORCS OU DE LISIERS «DÉNITRIFIÉS» ET RISQUES DE TRANSFERTS DE PHOSPHORE "AGRICOLE» DANS LES EAUX DE SURFACE ET LES NAPPES PHRÉATIQUES}

Le pouvoir épurateur du sol a souvent été, et continue d'être présenté comme une panacée qui permettrait d'éliminer nos déchets et, dans le cas présent, ceux des porcins (Catroux et al, 1974 ; Eliott et Coleman, 1988). Certes, le sol contient de très nombreuses populations bactériennes capables, après rejets de nos déchets organiques, de transformer leur carbone organique en $\mathrm{CO}_{2}$ et, dans la situation la plus favorable, leur azote organique uniquement en azote 
Tableau X. Effet à l'échelle annuelle des apports de lisiers sur le phosphore biodisponible et l'ambiance physicochimique dans la solution du sol de l'horizon 0-20 cm.

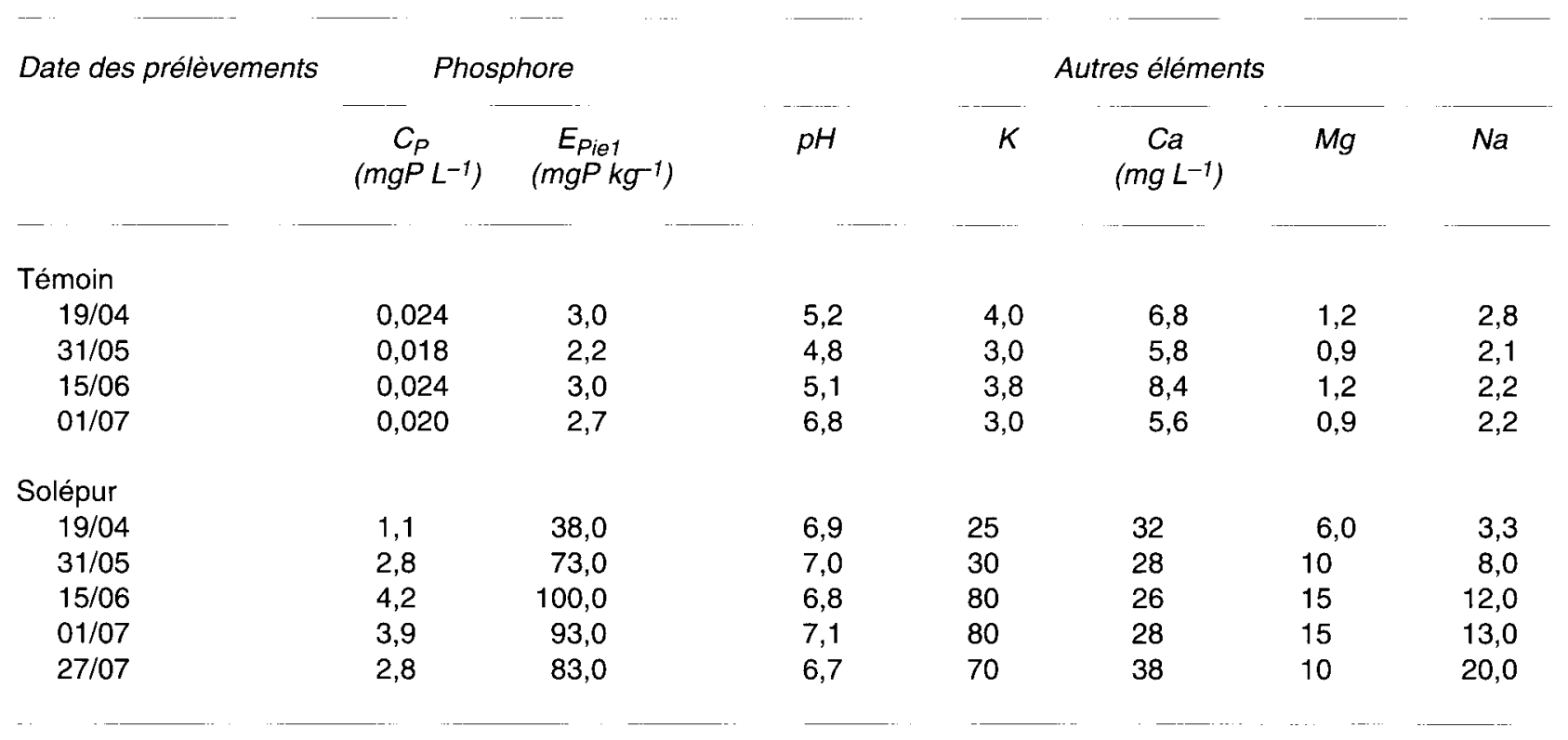

moléculaire, afin qu'il retourne dans l'atmosphère. Ce sont d'ailleurs ces propriétés microbiologiques des sols qui ont conduit à réaliser et faire fonctionner le dispositif Solépur.

Le phosphore, et le potassium, ne peuvent avoir le destin du carbone et de l'azote, aucun processus microbiologique ne permettant de s'en débarrasser. Les résultats présentés démontrent que, dans le sol utilisé, le phosphore des lisiers, ou celui des produits qui en sont dérivés par des processus d'ammonification puis de nitrification, doit être considéré comme à l'échelle pluriannuelle comme aussi biodisponible que celui des engrais phosphatés minéraux soluble-eau.

Le phosphore est l'élément déclenchant les processus d'eutrophisation des eaux de surface (Barroin, 1990), lorsque celles-ci récèlent suffisamment d'azote disponible, ce qui est toujours le cas. II faut cependant rappeler que les phosphates ne sont toxiques, ni pour l'homme, ni pour les animaux, ni pour les végétaux et que les effets néfastes attribués au phosphore ne sont qu'une conséquence indirecte de leur présence, en association avec de l'azote biodisponible, dans les milieux aquatiques (Sharpley et Withers, 1993).

Le phosphore étant une ressource non renouvelable (Sheldon, 1982), essentiellement utilisée pour la production agricole, il convient d'éviter son transfert vers les eaux où il serait exclu, pour des ères, du cycle agricole. Cette analyse conduit à recommander, sauf exception, de ne pas apporter, dans des sols suffisamment pour- vus, une quantité de phosphore supérieure à celle susceptible d'être prélevée par les cultures durant la rotation culturale (Anonyme, 1993), voire celle exportée par la récolte puisque le phosphore de résidus de culture enfouis possède une efficacité agronomique équivalente à celle des formes soluble-eau (Thibaud et al, 1988).

II conviendrait aussi de gérer le phosphore présent dans les lisiers, ou dans ses dérivés biologiques dont la teneur du phosphore est quatre à cinq fois plus élevée que celle des lisiers, selon la démarche la plus rationnelle possible. En raison de la densité élevée des élevages porcins en certains points du territoire, les sols recevant, et appelés à recevoir, des lisiers sont déjà généralement largement pourvus (Coppenet et al, 1993). Nos résultats font apparaître une migration dans les horizons successifs des sols. Dans le dispositif concerné, le pouvoir fixateur pour les ions phosphate croît très vite avec la profondeur : dans ce cas particulier les risques de transfert vers les nappes phréatiques semblent donc pratiquement exclus et l'érosion serait la voie majeure de transfert du phosphore vers les eaux de surface, qui sont précisément celles marquées par les processus d'eutrophisation. Toute particule de sol issue de parcelles ayant reçu des quantités importantes de lisier et tombant à l'eau sera source significative de phosphore biodisponible. Ce phosphore sera d'autant plus biodisponible pour les algues que la matière organique présente dans le lisier contient de l'azote et diminue le pouvoir fixateur des particules de sol. Cependant l'accroissement du pouvoir fixateur avec la pro- 
fondeur ne peut être généralisée à toutes les situations, quoiqu'elle soit probablement la plus fréquente. En effet, certains auteurs (Mozaffari et Sims, 1994 ; Simard et al, 1995) ont signalé, également dans des situations agricoles dominées par les pratiques d'élevage, la présence d'horizons $B$ moins fixateurs du phosphore que les horizons $A$. Cependant dans les situations décrites par ces auteurs la teneur des sables est souvent proche de $80 \%$, ce qui n'est certainement pas la situation la plus fréquente en Bretagne.

La logique agronomique conduirait donc à recommander d'épandre les lisiers de préférence dans des sols peu pourvus, mais surtout possédant un pouvoir fixateur, et un pouvoir tampon, les plus élevés possible. C'est uniquement dans ce cas que les particules entraînées dans les eaux de surface n'autoriseraient pas l'apparition de concentrations élevées de phosphore dans les eaux et, ipso facto, élimineraient les risques d'eutrophisation.

\section{CONCLUSION}

Les résultats présentés autorisent deux conclusions principales : I'une concerne un aspect méthodologique relatif aux techniques d'estimation en routine du phosphore assimilable dans des échantillons de terre prélevés dans des parcelles recevant, ou ayant reçu, du lisier ; l'autre porte sur le devenir, et les conséquences, d'apports de phosphore significativement très supérieurs aux quantités prélevées par les récoltes, que l'apport ait lieu sous forme de lisiers ou de phosphates minéraux.

Dans les sols ayant reçu des quantités de lisier significativement supérieures à celles exportées par les récoltes, la méthode Dyer utilisée en France dans les sols non calcaires surestime les quantités de phosphore considérées comme assimilables. Cette situation a pour origine l'hydrolyse des phytates de calcium-magnesium par l'acide citrique à $2 \%$. Ce résultat démontre encore que des formes de phosphore non assimilables au moment de leur application sur les terres peuvent être comptabilisées comme formes assimilables par la méthode Dyer. II illustre la difficulté qu'il peut y avoir à interpréter les quantités de phosphore extraites d'un sol par la méthode Dyer. En effet, sauf exception, un laboratoire recevant une terre pour analyse n'est pas en mesure de savoir si une parcelle a été amendée, ou non, par des déjections animales.

Les résultats permettent de généraliser quelques observations obtenues antérieurement dans un dispositif très différent, voire discutable (Fardeau et Guiraud, 1971). À l'échelle annuelle et dans les sols dont les $\mathrm{pH}$ sont supérieurs à 5,5 , le phosphore présent dans les déjections animales devient à l'échelle pluriannuelle aussi biodisponible (Lucerno et al, 1995) que le phosphore apporté par n'importe quel engrais phosphaté minéral, tels les phosphates d'ammonium, le superphosphate simple ou triple ou le phosphate bicalcique, dont la solubilité du phosphore, à l'instant de leur application sur le sol, est 100 à 1000 fois supérieure à celle du phosphore du sol. La présence des matières organiques du lisier contribue à diminuer le pouvoir fixateur des particules de sol. En conséquence, chaque fois que du phosphore est apporté sous forme de lisier en quantités très supérieures à celles exportées par les récoltes, la teneur du phosphore biodisponible dans le sol, et sa biodisponibilité, augmenteront. Les particules victimes de l'érosion et rejoignant les rivières seront des sources certaines de phosphore biodisponible pour les eaux de surface. De plus, dans ces situations, du phosphore migrera vers des horizons plus profonds que celui où il a été appliqué. Les conditions dans lesquelles ce phosphore pourra rejoindre la nappe phréatique dépendent principalement de la profondeur de la nappe et du pouvoir fixateur des horizons sous-jacents.

Enfin, l'addition de lisier à des sols accroît non seulement le contenu en éléments nutritifs majeurs, à savoir $\mathrm{N}, \mathrm{P}$ et $\mathrm{K}$, mais également le $\mathrm{pH}$ et les teneurs du calcium è du magnésium dans la solution du sol.

\section{REMERCIEMENTS}

Le suivi de la station pilote Solépur est réalisé grâce au soutien financier du conseil général du Finistère et du conseil régional de Bretagne (programme Bretagne Eau pure). Nous tenons également à remercier $\mathrm{M}$ Bertrand, le concepteur du projet Solépur, pour son sens de l'initiative.

\section{RÉFÉRENCES}

Anonyme (1993) Aide au diagnostic et à la prescription de la fertilisation phosphatée et potassique des grandes cultures. Comifer, Paris 
Barber SA (1984) Soil nutrient bioavailability. A mechanistic approach. John Wiley and Sons, New York

Barbier G, Bilaus J, Boniface R (1965) Contribution à l'étude de l'influence du $\mathrm{pH}$ sur la mobilité des ions phosphoriques. Ann Agron 16, 603-622

Barroin G (1990) La pollution des eaux par les phosphates. La Recherche 21, 620-627

Beckett PHT, White RE (1964) Studies on potentials of soils. Part III. The pool of inorganic phosphate. Plant Soil 21, 253-282

Bernal MP, Lax A, Roig A (1993a) The effect of pig slurry on exchangeable potassium in calcareous soils. Biol Fertil Soils 16, 169-172

Bernal MP, Roig A, Garcia D (1993b) Nutrient balances in calcareous soils after application of different rates of pig slurry. Soil Use Manage 9, 9-14

Boniface T, Trocme S (1988) Enseignements fournis par des esais de longue durée sur la fumure phosphatée et potassique. 2. Essais sur la fumure phosphatée. In : Phosphore et potassium dans les relations sol-plante : conséquence sur la fertilisation (L Gachon, ed), Inra, Paris, 279-302

Bosc M (1988) Enseignements fournis par des esais de longue durée sur la fumure phosphatée et potassique. 3. Essais sur la fumure potassique. In : Phosphore et potassium dans les relations sol-plante : conséquence sur la fertilisation (L Gachon, ed), Inra, Paris, 303-466

Bowman RA, Cole CV (1978a) Transformations of organic phosphorus substrates in soils as evaluated by $\mathrm{Na} \mathrm{HCO}_{3}$ extraction. Soil Sci 125, 49-54

Bowman RA, Cole CV (1978b) An explanatory method for fractionation of organic phosphorus from grassland soils. Soil Sci 125, 95-101

Bril J, Salomons W (1990) Chemical composition of animal manure: a modelling approach. Netherlands J Agric Sci 38, 333-351

Catroux G, Germon JC, Graffin P (1974) L'utilisation du sol comme système épurateur. Ann Agron 25, 179-193

Cheverry C (1994) La dégradation chimique des sols en Bretagne. Étude et Gestion des Sols 1, 7-21

Christie P (1987) Long term effects of slurry on grassland. In : Animal Manure on Grassland and Fodder Crops (HG Van Den Meer et al, eds), Martinus Nijhoff Publishers, Dordrecht, 301-304

Coppenet M (1974) L'épandage du lisier de porcherie. Ses conséquences agronomiques. Ann Agron 25, 403-423

Coppenet M (1985) Bilan des éléments fertilisants dans les exploitations d'élevage. Application à une fertilisation raisonnée. Techniques Agricoles 1315

Coppenet M (1988) Influence de la teneur du sol en $\mathrm{P}_{2} \mathrm{O}_{5}$ assimilable, de l'apport d'engrais phosphatés et de la hauteur des précipitations estivales sur la richesse en phosphore du ray-grass italien (Lolium multiflorum $\mathrm{L}$ ). In : Phosphore et potassium dans les relations sol-plante : conséquence sur la fertilisation (L Gachon, ed), Inra, Paris, 481-513
Coppenet M, Golven J, Simon JC, Le Corre L, Le Roy M (1993) Évolution chimique des sols en exploitations d'élevage intensif : exemple du Finistère. agronomie $13,77-83$

Cosgrove DJ (180) Inositol Phosphates. Their Chemistry, Biochemistry and Physiology. Elsevier, Amsterdam

Cromwell GL (1992) The biological availability of phosphorus in feedstuffs for pigs. Pigs News and Information 13, 75-78

Devloo O (1994) Nitrification de l'azote des lisiers de porcs dans un procédé de traitement par le sol. Suivi du pilote Solepur. Mémoire de troisième année de l'Enesad, Quétigny

Dighton J (1991) Acquisition of nutrients from organic resources by mycorrhizal autotrophic plants. Experientia 47, 362-369

Duval L (1988) Étude comparée de l'extraction du phosphore assimilable des sols de la Bretagne occidentale. In : Phosphore et potassium dans les relations sol-plante : conséquences sur la fertilisation (L Gachon, ed), Inra, Paris, 217-224

Eliott ET, Coleman DC (1988) Let the soil work for us. Ecol Bull 39, 23-42

Fardeau JC (1993) Le phosphore assimilable des sols : sa représentation par un modèle fonctionnel à plusieurs compartiments. agronomie 13, 317-331

Fardeau JC, Guiraud G (1971) Mobilité du phosphore d'un sol ayant reçu du fumier pendant 35 ans. $C R$ Acad Agri Fr 1598-1605

Fardeau JC, Dellile D, Abramovici C (1968) Utilisation de la phytine par les plantes. In : Isotopes and radiation in soil organic matter studies. AIEA/SM106-37, AIEA, Vienne, 555-565

Fardeau JC, Morel C, Boniface R (1988) Pourquoi choisir la méthode Olsen pour estimer le phosphore «assimilable» des sols ? agronomie 8, 577-584

Fardeau JC, Morel C, Boniface R (1991) Cinétiques de transfert des ions phosphate du sol vers la solution du sol : paramètres caractéristiques ? agronomie 11, 787-797

Frossard E (1985) Étude expérimentale de l'influence de composés organiques sur l'évolution des ions phosphate en sols ferralitiques. Thèse INPL, Nancy

Frossard E, Fardeau JC, Brossard M, Morel JL (1994) Soil isotopically exchangeable phosphorus: a comparison between $\mathrm{E}$ and $\mathrm{L}$ values. Soil Sci Soc Am J 58, 846-851

Gachon L (1977) Utilité d'un bon niveau de réserves phosphatées du sol. Phosph Agric 70, 27-33

Hartikainen $H$ (1991) Potential mobility of accumulated phosphorus in soil as estimated by indices of $Q / 1$ plots and by extractant. Soil Sci 152, 204-210

Hénin S (1990) Phosphate et environnement. $C R$ Acad Agri Fr 76, 25-28

Japenga J, Harmsen K (1990) Determination of mass balances and ionic balances in animal manure. Neth J Agric Sci 38, 353-367 
Judel GK, Gebauer WG, Mengel K (1985) Yield response and availability of various phosphate fertilizer types as estimated by EUF. Plant Soil 83, $107-116$

Juste C (1988) Appréciation de la mobilité et de la biodisponibilité des éléments en traces dans le sol. Sci Sol 26, 103-112

Kucey RMN, Janzen HH, Legget ME (1989) Microbially mediated increases in plant available phosphorus. Adv Agron 42, 198-228

Lucerno DW, Martens DC, McKenna JR, Starner DE (1995) Poultry litter effects on unmanaged pasture yield, nitrogen and phosphorus uptakes, and botanical composition. Comm Soil Sci Plant Anal 5-6

Martinez J (1994) Nutrients accumulation in soil from intensive pig slurry applications. In : Animal Waste Management. Proceedings of European Cooperative Research Network on Animal Waste Management. Seven Consultation (JE Hall, ed), FAO, Bad Zwischenahn, 129-135

Martinez J, Guiziou F (1995) Mise au point et évaluation de la faisabilité d'un procédé naturel de traitement du lisier de porcs par le sol. In : Journées Rech Porcine en France 27, 367-372

Mousain D (1989) Étude de la nutrition phosphatée de symbiotes ectomycorhiziens. Thèse doctorat d'État, Univ Sci Techn Languedoc

Morel C, Plenchette C (1994) Is the isotopically exchangeable phosphate of a loamy soil the plant available P? Plant Soil 158, 287-297

Mozaffari M, Sims JT (1994) Phosphorus availability and sorption in ana Atlantic coastal plain watershed dominated by animal-based agriculture. Soil $\mathrm{Sci}$ 157, 97-107

N'dayegamiye A, Cote D (1989) Effect of long term pig slurry and solid cattle manure application on soil chemical and biological properties. Can J Soil Sci $69,39-47$

Nirel PMV, Morel FMM (1990) Pitfalls of sequential extractions. Wat Rev 24, 1055-1056

Novozamsky I, Van Dijk D, Van der Lee JJ, Houba VJG (1993) Automated determination of trace amounts of phosphate in soil extracts using malachite green. Commun Soil Sci Plant Anal 24, 10651076

Oberson A (1993) Phosphordynamic in biologish und konventionell bewirschafteten Böden des DOKVersuchs. Dissert ETH 10119. Zurich

Oberson A, Fardeau JC, Besson JM, Sticher H (1993) Soil phosphorus dynamics in cropping systems managed according to conventional and biological agricultural methods. Biol Fertil Soils 16, 111-117
Pape JC (1970) Plaggen soils in the Netherlands. Geoderma 4, 229-255

Papini R, Spallaci P, Primavera F (1994) Forme di fosforo in diversi suoli trattati con liquami suini e loro evoluzione nel tempo. Agrochimica XXXVIII, 315207

Robinson JS, Sharpley AN, Smith SJ (1995) The effect of animal manure applications on the forms of soil phosphorus. In : Animal Waste and Land-Water Interface (K Steele, ed), CRC-Lewis publishers, Boca Raton, 43-48

Sharpley AN, Smith SJ (1995) Nitrogen and phopshorus forms in soil receiving manure. Soil Sci 159 , 253-258

Sharpley AN, Withers PJA (1994) The environmentally-sound management of agricultural phosphorus. Fert Res 29, 133-146

Sheldon R (1982) Les phosphates. Pour la science 3441

Simard RR, Cluis D, Gangbazo G, Beauchemin S (1995) Phosphorus status of forest and agricultural soils from a watershed of high animal density. $J$ Environ Qual 24, 1010-1017

Stevens RJ, Laughlin RJ, Logan HJ, Gracey HI (1988) The effect of large applications of pig slurry on the strength of soil under grass. Soil Use and Management 4, 57-59

Syers JK, Lu Ru-kun (1990) Inorganic reactions influencing phosphorus cycling in soils. In : Phosphorus Requirements for Sustainable Agriculture in Asia and Oceania; Inter Rice Res Inst; Manilla, the Phillippines, 191-198

Tran TS, N'dayegamiye A (1995) Long-term effects of fertilizers and manure application on the forms and availability of soil phosphorus. Can J Soil Sci 75, 281-285

Thibaud MC, Morel C, Fardeau JC (1988) Contribution of phosphorus issued from crop residues to plant nutrition. Soil Sci Plant Nutr 34, 481-491

Van Veldhoven P, Manaerts GP (1987) Inorganic and organic phosphate measurements in the nanomolar range. Anal Biochem 161, 45-48

Whitehead DC, Bristow AW, Pain BF (1989) The influence of some cattle and pig slurries on the uptake of nitrogen by ryegrass in relation to fractionation of the slurry N. Plant Soil 117, 111-120

Zhang YS, Ni WZ, Sun X (1993) Influence of organic manure on organic phosphorus fraction in soils. Pedosphere 3, 361-369 